
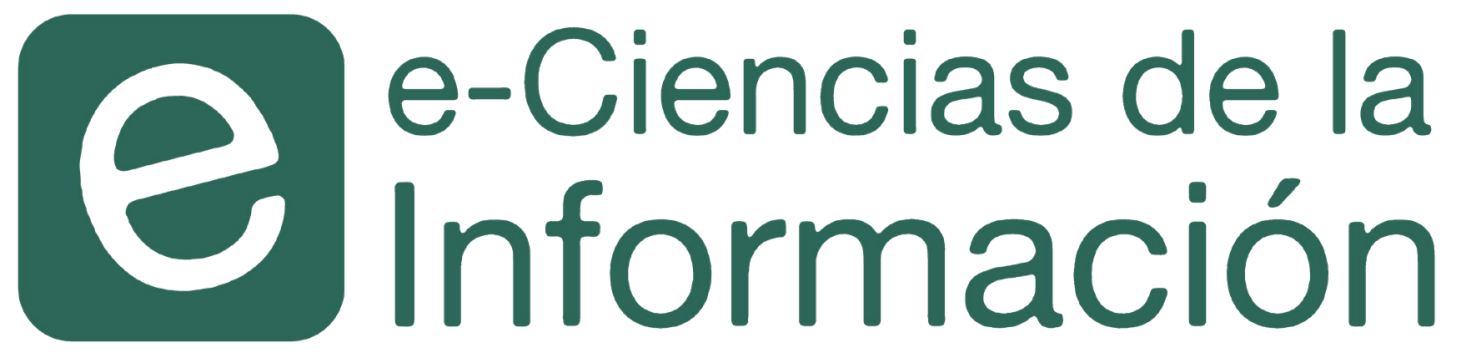

\title{
Influencia de la escuela, familia y medios de comunicación en el proceso de socialización política de estudiantes de primaria: Análisis en el contexto de Nuevo León, México \\ Carlos Muñiz Alma Rosa Saldierna Alondra Salazar América Batres
}

Recibido: 31/05/2019 | Corregido: 26/09/2019 | Aceptado:10/10/2019 DOI: $10.15517 /$ eci.v10i1.39776

e-Ciencias de la Información, volumen 10, número 1, Ene- Jun 2020

ISSN: 1649-4142

\section{다(1)\$()}

\section{¿Cómo citar este artículo?}

Muñiz, C., Saldierna, A. R., Salazar, A. y Batres, A. (2020). Influencia de la escuela, familia y medios de comunicación en el proceso de socialización política de estudiantes de primaria: Análisis en el contexto de Nuevo León, México. e-Ciencias de la Información, 10(1). Doi: 10.15517/eci.v10i1.39776 


\title{
Influencia de la escuela, familia y medios de comunicación en el proceso de socialización política de estudiantes
}

\author{
School, family and media influence on the process of po- \\ litical socialization of primary students: Analysis in the \\ context of Nuevo León, Mexico
}

\author{
Carlos Muñiz (D) ${ }^{1}$ \\ Alma Rosa Saldierna (iD ${ }^{2}$ \\ Alondra Salazar $\mathbb{D}^{3}$ \\ América Batres (1) 4
}

\section{Resumen:}

La socialización política implica el desarrollo de las concepciones del mundo político. Si bien la escuela es considerada un agente básico de socialización de los niños, otros actores también contribuyen a determinar su cultura política, como la familia o los medios de comunicación. Este artículo busca determinar el grado de influencia de estos agentes en el desarrollo del compromiso político de los niños. Para ello, se aplicó una encuesta a una muestra representativa $(\mathrm{N}=1009)$ de los estudiantes de primaria en el estado mexicano de Nuevo León. Se evaluó el nivel de atención a contenidos políticos en medios tradicionales y sociales, de conversación política en familia y de formación cívico-política recibida en la escuela, como variables independientes, y el interés político, el conocimiento político y el sentimiento de eficacia política, como dependientes. Los resultados muestran un impacto relevante de la formación cívico-política de la escuela y la atención a política en medios tradicionales sobre las orientaciones estudiadas. Sin embargo, la conversación política en familia y el seguimiento de política en medios sociales tuvieron una baja influencia. Se reflexiona acerca de las implicaciones que estas relaciones explicativas tienen en la configuración de la cultura política de los niños.

Palabras clave: Socialización política; cultura política; compromiso político; agentes socializadores; educación primaria

\section{ABSTRACT}

Political socialization implies the development of conceptions about the political world. Although school is considered the basic agent for the socialization of children, there are other actors that also contribute to their political culture, as the family or the media. This paper aims to determine

1Universidad Autónoma de Nuevo León, MÉXICO. carlos.munizm@uanl.mx http://orcid.org/0000-0002-9021-8198 2Universidad Autónoma de Nuevo León, MÉXICO. alma.saldiernas@uanl.mx http://orcid.org/0000-0003-1805-9740 3Universidad Autónoma de Nuevo León, MÉXICO. salazaraloh@gmail.com http://orcid.org/0000-0002-8987-3873 4Universidad Autónoma de Nuevo León, MÉXICO. americabatresc@gmail.com https://orcid.org/0000-0002-3870-1908 
the degree of influence of these agents in the crystallization of children's political engagement. To do this, a survey was carried out to a representative sample $(N=1009)$ of primary school students in the Mexican state of Nuevo León. The level of attention to political content in traditional and social media, conversation among family, and civic-political education received in the school were used as independent variables. Political interest, political knowledge and political efficacy were evaluated as dependent ones. Findings show a relevant effect from the civic-political education in the school and the political attention in traditional media on the political orientations studied. However, conversation among family, and political attention in social media had a low influence. The implications that these explanatory relationships have in the configuration of the children's political culture are discussed.

Key words: Political socialization; political culture; political engagement; socializing agents; primary education

\section{Introducción}

La socialización política es el término que se ha empleado para explicar el proceso mediante el cual los individuos desarrollan concepciones de sí mismos y su mundo, así como del mundo político que les rodea (Huerta y García, 2008). Atendiendo a la literatura existente, habitualmente se considera que un ciudadano cuenta con una buena socialización en el terreno político cuando cuenta con un buen conocimiento sobre política, que se plasma en un conjunto de esquemas amplio y variado que constituyen una adecuada cultura política (Sears y Valentino, 1997), lo que cristaliza en el desarrollo de actitudes comprometidas hacia los aspectos políticos importantes del día a día (Arens y Watermann, 2017; Delli Carpini, 2004). Si bien se ha considerado que el papel de la escuela como agente socializador es decisivo en estadios tempranos de desarrollo del individuo, hay otros actores que también contribuyen en la construcción de la cultura política entre los ciudadanos.

En este sentido, tanto la familia como los medios también constituyen agentes poderosos en la socialización política de los niños. En el caso concreto de los medios, se ha observado que éstos pueden tener una influencia significativa en las actitudes políticas de los individuos (Chaffee, Ward, y Tipton, 1970) en estadios iniciales de desarrollo (Rodríguez y Muñiz, 2009). Asimismo, también se considera que pueden contribuir a que los individuos desarrollen más interés y aumenten sus niveles de conocimiento y, de manera indirecta, aumente su subsecuente participación en cuestiones de política (Echeverría y Meyer, 2017; Huerta, Bañuelos, Rodríguez, Luz, y Gómez, 2006; Lee, Shah y McLeod (2012). Una realidad que también se ha observado con respecto al consumo de los contenidos políticos transmitidos a través de medios online (Kruikemeier y Shehata, 2017; Muralidharan y Sung, 2015).

Tomando todo ello como referencia, el presente artículo se plantea como objetivo analizar el papel ejercido por el sistema educativo en comparación a otros agentes socializadores, como la familia o los medios de comunicación, en la activación y fortalecimiento de una cultura política democrática entre los estudiantes. En este sentido, se busca determinar el peso de los diferentes actores que pueden intervenir en el proceso de socialización política temprana de los ciudadanos, en este caso niños estudiantes de educación básica que tienen una cultura política sostenida por dichos participantes. 
Más en concreto, se trabaja con cuatro dimensiones que determinan en buena parte el compromiso democrático de los ciudadanos, plasmado en el mantenimiento de una cultura política adecuada.

Así, se evalúa la influencia de los agentes socializadores sobre el interés hacia la política, que determina el compromiso afectivo de la ciudadanía con los asuntos públicos; el conocimiento político, que determina el nivel de sofisticación política de los ciudadanos en su comprensión sobre los asuntos públicos; y la eficacia política interna y externa, que determina la percepción ciudadana de formar parte del sistema y, por tanto, de contribuir a su desarrollo a través de sus acciones y actividades políticas.

\section{Marco teórico}

\subsection{La socialización política en la configuración de la cultura política}

La cultura política, entendida como un conjunto de actitudes, creencias, opiniones y normas que genera en el individuo una tendencia u orientación hacia la acción política y participación democrática, constituye un resultado de diferentes procesos de socialización política del ciudadano, en la que intervienen distintos actores o agentes a lo largo de su vida (Echeverría y Meyer, 2017). En la construcción de esta cultura política democrática, la socialización política constituye un factor fundamental (Mayer y Schmidt, 2004), puesto que es el proceso mediante el que se consolidan esos conocimientos, valores y actitudes que van a configurar la ciudadanía (Rodríguez y Muñiz, 2009; Stojnic, 2015). Este proceso comienza desde edades tan tempranas como los 5 años y se extiende hasta la adolescencia, cuando se comienzan a desarrollar creencias políticas y sociales sobre aspectos como la autoridad, la propiedad, la toma de decisiones, los símbolos políticos o las instituciones políticas, así como la confianza y creencias hacia las mismas (Delli Carpini, 2004).

La socialización política es el término que se ha empleado para explicar el proceso mediante el cual los individuos desarrollan concepciones de símismos y su mundo, así como del mundo político (Huerta y García, 2008). Es decir, un proceso de aprendizaje a través del cual las normas y comportamientos políticos aceptables dentro de un sistema político concreto son transmitidos desde una generación a otra (Mayer y Schmidt, 2004). Y, en particular, el proceso mediante el cual los adolescentes adquieren actitudes, cogniciones y comportamientos relacionados con su medio ambiente político (Arens y Watermann, 2017; Muralidharan y Sung, 2016). Así, la socialización mantiene o cambia la cultura política de los ciudadanos a través del desarrollo de orientaciones políticas democráticas, como pueden ser actitudes, creencias y conductas relativas al sistema político del cual forma parte el niño o adolescente (Dahl y Abdelzadeh, 2017).

Se considera que un individuo estará bien socializado si tiene buena información y ésta cristaliza en actitudes hacia los aspectos políticos importantes del día a día (Sears y Valentino, 1997). Sin embargo, el proceso que lleva a esta materialización se puede producir en diferentes momentos (Berger y Luckman, 1983): existe una socialización primaria presente en los primeros años de vida, construida en el seno familiar, educativo y mediático, 
y una socialización secundaria que se forma con base en la relación de los sujetos y la experiencia que adquiere el individuo. Sin duda, es la socialización primaria la que marcará en gran medida el nivel y amplitud de conocimientos, creencias políticas y tendencia hacia la participación futura en asuntos políticos de los niños y adolescentes, configurando sus representaciones sobre qué implica ser ciudadano y constituye la ciudadanía (Cullingford, 1992).

A través de ella se genera un compromiso político en estos futuros ciudadanos con los valores democráticos, que les permitirá estar atentos y vigilantes a los actos del gobierno y buscar la manera de incidir en éste. Si bien es complicado definir unívocamente qué constituye el compromiso cívico, autores como Delli Carpini (2004) señalan que éste implica el desarrollo de valores democráticos (como la existencia de una eficacia política aceptable). También la generación de actitudes sólidas sobre el mundo político y social (como la orientación por conocer los acontecimientos políticos y sociales manifestada en el interés político), la posesión de opiniones consistentes e informadas sobre temas de debate público (es decir, mantener un conocimiento político relevante) $y$, además, el involucramiento a través de acciones que busquen la mejora de la calidad del sistema. Estas orientaciones configuran las dimensiones afectiva, cognitiva y conductual que normalmente vistos como los factores determinantes de la cultura política ciudadana (Echeverría y Meyer, 2017) y que derivan del proceso de socialización política (Arens y Watermann, 2017).

Pasando al detalle de cada uno de estos elementos, el interés en la política ha sido descrito como el principal predictor para determinar el comportamiento político (Prior, 2010), en tanto que es el desencadenante del proceso que lleva a la búsqueda de información política, a su procesamiento y empleo para la toma de decisiones. Es, por tanto, una orientación afectiva manifestada en la motivación para informarse sobre los asuntos públicos y políticos (Delli Carpini, 2004) e involucrarse en política, lo que posibilita el proceso de aprendizaje y de conformación del conocimiento político factual (Dahl y Abdelzadeh, 2017). Este conocimiento habitualmente ha sido definido como el conjunto de nociones objetivables que los ciudadanos mantienen acerca del sistema político, de cómo está estructurado, de cómo funciona o cuáles son sus reglas de juego, de quiénes son los principales actores protagonistas dentro del mismo y qué es lo que éstos hacen (Delli Carpini, 2004).

Pero un compromiso cívico efectivo requiere del involucramiento del ciudadano en el desarrollo del sistema, a través de orientaciones previas, como por ejemplo el sentimiento de eficacia política (Delli Carpini, 2004). Este sentimiento remite a la percepción que tienen los individuos de poder influir en el proceso político (Arens y Watermann, 2017). Normalmente se diferencia entre eficacia interna, que implica una autopercepción de estar preparado y tener capacidad para entender lo que ocurre en el sistema, frente a la eficacia externa, que remite a la percepción sobre la responsabilidad del sistema y sus gobernantes para con las demandas de los ciudadanos (Arens y Watermann, 2017). Este sentimiento se comienza a desarrollar en una etapa temprana del individuo y marca su probabilidad de compromiso futuro (Delli Carpini, 2004), motivo por el cual se ha visto como una medida indirecta de la participación ciudadana (Miron y Bryant, 2007).

Es decir, se asume que aquellos ciudadanos verdaderamente comprometidos con la democracia son los que están "más interesados en política y más conocedores son de los asuntos políticos, son más eficaces políticamente, más tolerantes y confiables y están involucrados más activamente en política 
(Dahl y Abdelzadeh, 2017, p. 1252). Se observa, por tanto, que esta medida de eficacia política puede ser adecuada para medir de forma indirecta el involucramiento de los estudiantes, asumiendo que no existe como tal la participación cívica del niño, ya que no es considerado todavía ciudadano con plena capacidad para defender sus derechos políticos (Imhoff y Brussino, 2013).

Ahora bien, para que se genere esa cultura política, es necesario el concurso de diferentes factores de socialización entre los individuos. Al respecto, Muralidharan y Sung (2016) recuerdan que en la socialización política se presentan cuatro componentes principales: variables estructurales sociales, agentes de socialización, resultados mentales y resultados de comportamiento. Todos ellos se presentarán en la medida en que los niños y adolescentes interactúen con los diferentes agentes socializadores, como son la familia, los amigos, los maestros o los medios de comunicación (Echeverría y Meyer, 2017).

\subsection{Principales agentes de la socialización política}

Sin duda, uno de los principales agentes socializadores en estadios tempranos de desarrollo del individuo es la escuela y sus maestros (Stojnic, 2015). La educación en la escuela, como herramienta de formación por excelencia que tiene el estado, incide en la construcción de una cultura democrática a través de la generación de civismo, es decir, enseñando a los niños cómo actuar en la colectividad (Echeverría y Meyer, 2017). De esta manera, la educación tiene en sus manos comunicar, compartir y vivenciar principios democráticos y estrategias de aprendizaje para que se edifiquen pilares sólidos en la democracia de un país (Huerta y García, 2008). Por ello, el sistema educativo tiene la obligación de formar una conciencia cívica en los ciudadanos, además de ser dinámico y promover el diálogo entre los estudiantes, el cual fortalece y perfecciona el desarrollo de actitudes favorables hacia la democracia; algo que se puede lograr a través de la inclusión de mecanismos que favorezcan la participación de los estudiantes dentro de las escuelas (Imhoff y Brussino, 2013).

Se observa, por tanto, cómo la formación ciudadana debe empezar desde las aulas, ya que, dependiendo del modelo de ciudadano que se forme en ellas, así será el tipo de sociedad con la que se contará en el futuro (Eveland, McLeod, y Horowitz, 1998). La educación permite que los ciudadanos desarrollen las competencias necesarias para vivir en democracia (Echeverría y Meyer, 2017; Stojnic). Por tanto, la educación es un eslabón clave para la conformación de una democracia estable, mediante la formación de los ciudadanos, algo que se realiza en el tiempo y en el espacio (Arens y Watermann, 2017). Gracias a ello, se considera que cuando un niño termina la primaria, éste habrá completado el desarrollo de las orientaciones afectivas y adquirido las competencias cognitivas relativas a la formación de ciudadanía que definirán su comportamiento político futuro (Eveland et al., 1998).

Aunque la escuela constituye un actor crucial en la formación política del ciudadano, son diversos los agentes que contribuyen a la generación de este proceso de socialización política. Así, durante la socialización primaria la familia quizá se presenta como otro agente por excelencia, por ser el primer régimen político en el que se involucran los niños (Mayer y Schmidt, 2004), 
incidiendo de manera clara en la formación de sus habilidades cívicas y orientaciones hacia la política (Echeverría y Meyer, 2017). Durante la niñez, la persona adquiere ciertas nociones sociales y políticas precedentes de la familia, mismas que son utilizadas para construir los esquemas mentales de los que se servirán para comprender las relaciones de poder (Cullingford, 1992).

Por tanto, el capital cultural aportado por los padres y familiares es un condicionante clave para la conformación de la cultura política del ciudadano. Autores como Huerta, Bañuelos, Rodríguez, Luz y Gómez (2006) recuerdan cómo, mientras la escuela hace a los niños conscientes de los asuntos políticos, en la familia se forma la afiliación a partidos y prácticas tendentes a la participación. Este papel de la familia en el proceso de socialización política ha sido abordado por numerosos autores (Cullingford, 1992; Huerta y García, 2008; Huerta et al., 2006; López Gómez, 2003; Mayer y Schmidt, 2004). Al respecto, Mayer y Schmidt (2004) detectaron que lo que más impacta en el interés de los niños era la conversación con el padre y con la madre.

Por su parte, los medios contribuyen a que la población aumente sus niveles de conocimiento y la subsecuente participación en cuestiones de política (Echeverría y Meyer, 2017; Huerta et al., 2006), algo que también se ha observado respecto a los contenidos políticos transmitidos a través de Internet (Kruikemeier y Shehata, 2017; Muralidharan y Sung, 2016). En este sentido, los medios son un agente poderoso en la socialización política, teniendo una influencia significativa en las actitudes políticas de los individuos (Chaffee, Ward, y Tipton, 1970) en estadios iniciales de desarrollo (Rodríguez y Muñiz, 2009). No en vano, y como señala Delli Carpini (2004), los medios proveen buena parte de la "materia prima" necesaria para establecer las creencias, actitudes y esquemas políticos y sociales con que cuentan las personas (p. 408). Así lo pone de manifiesto el estudio realizado entre jóvenes de 12 y 17 años por Lee et al. (2012). Los resultados de autores permitieron determinar que los medios de comunicación cumplían un fuerte efecto en la socialización de los jóvenes con respecto a la vida política, pues su uso como fuentes de información tenía un peso fundamental en la generación de un compromiso cívico futuro.

\subsection{El estudio de la socialización política en México}

A pesar del importante desarrollo que esta línea de investigación tiene a nivel internacional, son relativamente pocos los estudios de socialización política desarrollados en México (Del Río, 2008; Huerta y García, 2008). Además, buena parte de estos resultados provienen de estudios en ámbitos urbanos, relegando el estudio de la socialización política a los contextos rurales. Todo ello a pesar de que la propia Secretaría de Educación Pública (SEP) del Gobierno de México aprobó en 2008 el Programa Integral de Formación Cívica y Ética (PIFCyE) para educación primaria. Con el mismo, se buscaba fortalecer una cultura política democrática en los niños, entendida ésta "como la participación activa en asuntos de interés colectivo para la construcción de formas de vida incluyentes, equitativas, interculturales y solidarias, que enriquezcan el sentido de pertenencia a su comunidad, su país y la humanidad" (p. 8).

Desde el estudio seminal realizado por Segovia (1975), otros autores han 
seguido esta línea de investigación para diagnosticar el estado de formación de los ciudadanos, tratando de identificar si estos sujetos son críticos de los procesos que se dan en la esfera pública. Destaca el estudio de Nateras (2007), quien encontró que la exposición a información política en medios era baja (49.3\%) entre estudiantes de primaria y secundaria y que el grado de interés e implicación política era más bien bajo en ese grupo etario. Además, detectó diferencias entre tipos de escuelas, presentando los de las privadas mayor acceso a la información política y mayor interés hacia la política. Sin embargo, Huerta y García (2008) encontraron que los niños con mayor disposición para participar eran los de la escuela pública.

Por su parte, Tapia (2003) al integrar en su observación a los medios de comunicación como reforzadores de las representaciones sociales y políticas, encontró que los niños que leían periódico y veían la televisión tenían más información política. Asimismo, Fernández Poncela (2009) detectó en un estudio comparado entre México y España que los jóvenes de entre 12 y 29 años estaban distanciados del sistema político, con desinterés y apatía, pero no se mostraban contrarios a los valores democráticos. Más recientemente, Morales (2014) ha documentado desafección hacia la política entre jóvenes de primaria y secundaria, siendo los estudiantes de escuelas privadas quienes hablan más de política, participan más en pláticas sobre temas de política, se interesan más en política y tienen una mayor percepción de los políticos como malos.

Dentro de los estudios en ámbitos subnacionales de México, destaca el estudio comparativo de López Gómez (2003) sobre procesos de socialización política en los niños de Juchitán (Oaxaca) y Monterrey (Nuevo León). El estudio detalla cómo los niños más envueltos en las dinámicas políticas de su entorno eran aquellos con los que sus padres se hallaban más involucrados en la esfera política, algo que se producía en mayor medida en el sur de la República. En el caso del norte de México, los estudios de Huerta et al. (2006) y de Huerta y García (2008) encontraron que los estudiantes de primaria del estado de Nuevo León tenían, en términos generales, una percepción de la política más negativa que positiva. Los autores también señalaron el gran peso que tenía la televisión cómo agente socializador, pero siempre después de otras como los maestros y, sobre todo, la familia. Tanto la escuela como la familia tenían un peso predominante entre estudiantes de escuelas privadas, frente a los de las públicas, donde otros agentes socializadores como los medios poseían mayor presencia. Tomando en cuenta la evidencia existente, se plantearon las siguientes preguntas de investigación que se espera resolver con los datos del estudio:

PI1: ¿En qué medida influyó cada agente socializador sobre el nivel de interés hacia la política mantenido por los participantes?

PI2: ¿En qué medida influyó cada agente socializador sobre el nivel de conocimiento político mantenido por los participantes?

PI3: ¿En qué medida influyó cada agente socializador sobre el nivel de eficacia política interna mantenido por los participantes?

PI4: ¿En qué medida influyó cada agente socializador sobre el nivel de eficacia política externa mantenido por los participantes? 


\section{Método}

\subsection{Enfoque}

Para la realización del estudio, se desarrolló una metodología de corte cuantitativa, consistente en la aplicación de una encuesta descriptiva con una muestra representativa de los estudiantes de quinto y sexto grado de educación primaria de las diferentes zonas del estado mexicano de Nuevo León. En este sentido, los datos obtenidos a partir de su levantamiento se trabajaron desde un acercamiento correlacional, con alcance explicativo, para determinar la influencia de los diferentes agentes socializadores en el desarrollo de diferentes actitudes políticas de los niños.

\subsection{Población de estudio}

Se cálculo una muestra del total de estudiantes de primaria en $5^{\circ}$ y $6^{\circ}$ grado que a la fecha de realización del estudio se encontraban estudiando en escuelas del estado mexicano de Nuevo León. A partir del total de estudiantes en el semestre agosto-diciembre de $2017(\mathrm{~N}=181,783)$, se seleccionó una muestra probabilística de 1,009 encuestados con un margen de error de +/$2.8 \%$ y un $97.8 \%$ de nivel de confianza. Con el objetivo de que la muestra fuera lo más representativa posible, se ajustó la composición para que representara tanto a la Zona Metropolitana de Monterrey $(n=147,027)$ y al resto de zonas del estado $(n=34,756)$. Para obtener la muestra, se realizó un muestreo probabilístico por conglomerados, trabajando con las zonas del estado como primer conglomerado, el tipo de escuelas (pública vs. privada) como segundo y por último, el salón dentro de la escuela como el tercero.

En este sentido, del total de centros que imparten enseñanza primaria en el estado, se seleccionaron al azar 53 escuelas participantes. Para dar la posibilidad de que tanto la muestra de la Zona Metropolitana de Monterrey (ZMM) como la del resto de zonas del estado fueran representativas, se determinó un número de 32 centros en la ZMM y 21 de otras zonas en las que se debían aplicar las encuestas. Además, y atendiendo a que del total de estudiantes de primaria del estado el $87.97 \%$ asistían a centros públicos y el $12.03 \%$ restante lo hacían a centros privados, se determinó que era necesario aplicar la encuesta en 48 centros públicos y 5 escuelas privadas. A partir del censo de escuelas de primaria del estado, se procedió a invitar a las diferentes escuelas para participar, contando finalmente con las accedieron a colaborar en el estudio y que cumplieran con el requisito de contar con al menos un grupo de cuarto y otro de quinto grado y con un mínimo de 25 estudiantes por grupo. El trabajo de campo fue realizado entre abril y mayo de 2017. Se contó para ello con la colaboración de los diferentes integrantes del grupo de investigación, así como de diversos estudiantes de licenciatura y posgrado de la Facultad de Ciencias Políticas y Relacionales Internacionales de la Universidad Autónoma de Nuevo León.

Previamente a la aplicación de los cuestionarios, las personas encuestadoras recibieron capacitación por parte del quipo investigador y se realizó una 
prueba piloto para validar las escalas utilizadas y comprobar la pertinencia de los reactivos utilizados en el cuestionario. Una vez en las escuelas participantes en el estudio, los investigadores procedieron a seleccionar al azar un aula con estudiantes de quinto o sexto grado de primaria -en caso de que hubiera más de una- y se aplicó la encuesta a todos los estudiantes del salón. Al tratarse de un cuestionario aplicado a niños, se solicitó la autorización previa de la autoridad educativa correspondiente en cada plantel y de los padres de familia. Cada uno de los encuestadores tuvo que estar plenamente identificado por la dirección del centro para poder acceder a las instalaciones, así como por el profesor titular de cada grupo asignado, que estuvo presente al momento de la aplicación del cuestionario.

\subsection{Técnicas de recolección}

La recolección de la información se realizó mediante la aplicación de un cuestionario que contemplaba el empleo de las siguientes escalas de medición (Ver documento complementario para conocer la redacción concreta de cada escala de medición):

\subsubsection{Atención a política en medios tradicionales:}

Con el objetivo de medir el consumo de contenidos de política realizado por los estudiantes en medios tradicionales, como son televisión, radio o prensa escrita (Kruikemeier y Shehata, 2017), se les preguntó en qué medida ellos y su familia se enteraban habitualmente de política a través de contenidos como noticias nacionales, noticias internacionales, noticias locales, programas tradicionales de política y/o programas de sátira o humor consumidos en los medios de comunicación convencionales. Para ello, se utilizó una escala Likert que oscilaba entre nada (1) y bastante (5). Se obtuvo una buena consistencia interna en la escala $(\alpha=.87)^{5}$.

\subsubsection{Atención a política en medios sociales:}

Asimismo, se buscó determinar el nivel de consumo de contenidos de política realizado a través de medios sociales. Para ello, se preguntó a los participantes en qué medida ellos y sus familias obtenían información sobre política navegando por webs en Internet y mediante el acceso y uso de redes sociales. En este sentido, se utilizó una escala Likert que oscilaba entre nada (1) y bastante (5). Se obtuvo una buena consistencia interna en la escala $(\text { Coeficiente de Spearman-Brown }=.73)^{6}$.

\footnotetext{
${ }^{5}$ La prueba Alfa de Cronbach mide el grado de congruencia que se tiene al medir el concepto que describe la escala multi-ítem, es decir, hasta qué punto los diferentes reactivos que componen la escala miden una misma característica o atributo. Se asume que valores de Alfa superiores a .70 determinan buena fiabilidad o consistencia interna de la escala (Igartua, 2006).

${ }^{6}$ El Coeficiente de Spearman-Brown aporta el grado de fiabilidad, similar al valor alfa de Cronbach, cuando se trabaja con escalas compuestas únicamente por dos reactivos.
} 


\subsubsection{Conversación política en familia:}

Por otra parte, se preguntó a los participantes en qué medida hablaban sobre política con familiares. Para ello, se utilizó una escala de Likert de nada (1) a bastante (5) a partir de la que debían contestar el nivel de plática política con su padre, madre, abuelos, hermanos y otros familiares como sus primos. Se obtuvo una buena consistencia interna en la escala $(\alpha=.80)$.

\subsubsection{Formación cívico-política en la escuela:}

Finalmente, se preguntó a los participantes en qué medida consideraban (escala de 1 = nada a 5 = bastante) que en su escuela estaban expuestos a mecanismos participativos (por ejemplo: elecciones de representante de grupo), si tenían un órgano representativo de los estudiantes en asuntos escolares, se promovía participar en asambleas de aula y si sus profesores les enseñaban contenidos democráticos o promovían valores democráticos en el aula. Para crear esta escala, se trabajó con algunas propuestas previas, como los reactivos para medir la participación activa real de Imhoff y Brussino (2013) o la exposición a contenidos democráticos de Stojnic (2015). La escala resultante logró una buena consistencia interna $(\alpha=.75)$.

\subsubsection{Interés político:}

Con el objetivo de medir el nivel de compromiso afectivo de los participantes con la política, se les preguntó en qué medida estaban interesados en la política, mediante una escala de Likert que oscilaba entre nada (1) y bastante (5). Para realizar la pregunta, se incluyó el tema de política entre otros varios, con el objetivo de reducir posibles sesgos negativos hacia la temática.

\subsubsection{Conocimiento político factual:}

Asimismo, se midió el compromiso cognitivo de los participantes con la política, a partir de una batería de diferentes preguntas que permitieran medir el nivel de conocimiento político factual de los mismos, es decir, su comprensión de reglas y actores del sistema. En concreto, se les hicieron 15 preguntas sobre conocimiento general acerca de gobernantes (e.g. nombre del Gobernador), conocimiento del sistema (e.g. ¿cuáles son los tres poderes del estado?) y reconocimiento de actores (e.g. ¿es político o no Andrés Manuel López Obrador?) Las respuestas se recodificaron en acierto (1) o fallo (0) y se sumaron las variables para crear una escala $(r 20=.69)^{7}$.

\footnotetext{
${ }^{7}$ La Fórmula 20 de Kuder y Richardson (KR-20) es similar a la Alfa de Cronbach, pero se utiliza para determinar la consistencia interna de variables medidas de forma dicotómica ( 1 = sí, 0 = no).
} 


\subsubsection{Sentimiento de eficacia política interna:}

Por otra parte, se creó una escala a partir de algunas propuestas de autores previos (Imhoff y Brussino, 2013; Segovia, 2001), para medir el grado de acuerdo con una serie de afirmaciones que reflejaran eficacia política interna de los estudiantes. En concreto, se les cuestionó en qué medida, entre nada (1) y bastante (5) estaban de acuerdo con lo siguiente:"me gustaría involucrarme en la política, cuando sea grande", "me gustaría ser presidente o gobernante cuando sea grande", "me gustaría unirme con otros para mejorar el país", "para los políticos los niños son importantes", "Ios niños tienen derecho a participar en política" y "votar es útil". Aunque la consistencia interna de la escala no fue alta, si fue aceptable $(a=.65)$. Si bien la consistencia es inferior al límite tradicionalmente aceptado en ciencias sociales (Igartua, 2006), se trabajará con la escala al ser un instrumento elaborado expresamente para el estudio, si bien deberá ser revisado para futuras investigaciones.

\subsubsection{Sentimiento de eficacia política externa:}

Asimismo, se creó una escala para medir el grado de eficacia política externa de los participantes, es decir, en las instituciones y actores de autoridad en su entorno. Para ello, se trabajó con las siguientes afirmaciones: "como niño tengo derechos", "mis papás me permiten exponer mis ideas", "mis maestros me permiten exponer mis ideas", "Ios maestros deben escuchar a sus estudiantes respecto al mejoramiento de la escuela" y "mis papás toman mi opinión en cuenta". En todos los casos se cuestionó el grado de acuerdo con cada una de ellas, en una escala Likert que oscilaba entre totalmente de acuerdo (1) y totalmente en desacuerdo (5). La escala resultante logró una buena consistencia interna $(a=.76)$.

\subsubsection{Variables de control:}

Junto a los reactivos que constituirían las variables independientes y dependientes del estudio, se realizó una serie de preguntas a los participantes, cuyas respuestas serían contraladas en los modelos aplicados. En este sentido, se preguntó por el sexo del participante ( $1=$ niño; $2=$ niña) y la edad de la participante expresada en años. También se les pidió que indicaran, en caso de saberlo o recordarlo, el nivel de estudios que tenía su padre y madre ( $1=$ Sin estudios; 2 = Primaria; 3 = Secundaria; 4 = Preparatoria; 5 = Universidad; 6 $=$ Maestría; $7=$ Doctorado; $99=$ No sabe). Por otra parte, a la hora de capturar los datos se registró si el estudiante pertenecía a una escuela pública (1) o privada (2), en qué zona del estado se encontraba la escuela ( $1=Z \mathrm{ZMM} ; 2=$ Resto del estado) y en qué grado de estudios se encontraba el estudiante ( 1 = quinto; 2 = sexto).

\subsection{Procesamiento de análisis}

Una vez terminado el trabajo de campo, consistente en aplicar la encuesta en las diferentes escuelas participantes, un grupo de colaboradores 
de posgrado de la misma Facultad participaron en la captura de los cuestionarios y depuración de la base de datos. Esta tarea de captura y depuración concluyó en el mes de julio de 2017, procediéndose después a crear las escalas relativas a las variables independientes y dependientes del estudio, cuya fiabilidad interna fue validada a partir de la prueba estadística Alfa de Cronbach explicada en el punto anterior. Por su parte, las diferentes preguntas de investigación establecidas, relativas al chequeo del modelo que se ajustaba mejor para explicar cada una de las variables dependientes, fueron respondidas a partir de la aplicación de diferentes regresiones lineales múltiples, con método introducir, que permiten contrastar el impacto de diferentes variables independientes en la dependiente. El nivel de significancia estadística aplicada fue del $5 \%(a=.05)$, habitualmente utilizado en ciencias sociales (Igartua, 2006). Para todos estos procedimientos, se trabajó con el paquete estadístico SPSS versión 22.0.

\section{Análisis y resultados}

Como primer paso dentro del análisis de los datos, se procedió a describir la muestra utilizada a partir del análisis descriptivo de las diferentes variables que serían posteriormente controladas en los modelos explicativos evaluados. En primer lugar, se determinó que el $49.6 \%$ de los participantes eran mujeres (n $=500$ ), representando el $50.4 \%$ restante a los hombres. La muestra presentó edades comprendidas entre los 10 y los 13 años $(M=11, D E=0.72)$. En cuanto al grado de estudios al que pertenecían los participantes del estudio, el $68.4 \%$ estudiaba $6^{\circ}$ grado de educación primaria $(n=690)$, perteneciendo el $31.6 \%$ a quinto grado de primaria. Además, el $89.5 \%$ de la muestra ( $n=$ 903) estudiaba en escuelas públicas, mientras que es $10.5 \%$ restante lo había en privadas. La mayoría de los participantes estudiaban en escuelas de la ZMM $(83.7 \%, n=845)$, perteneciendo el $16.3 \%$ restante a escuelas de zonas rurales. Finalmente, los participantes declararon, en su mayoría, qué padres contaban con algún nivel de estudios. Así, el 23\% señaló que las madres ( $n=$ 233) tenían al menos cumplida secundaria, mientras que el 19\% señaló que sus padres $(n=190)$ también contaban con estos estudios. Este nivel fue el que se presentó en mayor medida para ambos padres de los participantes.

Con el objetivo de determinar los factores explicativos de las diferentes variables constitutivas de la cultura política analizadas, y así poder dar respuesta a las cuatro preguntas de investigación planteadas en el estudio, se realizaron diferentes regresiones lineales múltiples con cada una de las variables como dependientes o criterio. Se tomó esta decisión debido a que esta prueba permite "evaluar las relaciones de dependencia entre los valores de una única variable dependiente (...) y los correspondientes a dos o más variables independientes (Igartua, 2006, p. 664). En un primer bloque de cada regresión se introdujeron las variables de control, determinadas por el sexo y edad del participante, nivel de estudios de la madre y del padre reportado, tipo de escuela a la que asiste el participante, área del estado donde se encuentra la escuela (ZMM frente al resto) y grado escolar del participante (quinto o sexto grado). Ello para poder determinar una medición más real acerca del peso de cada agente socializador en cada uno de los elementos de la cultura política.

En cuanto al análisis del interés hacia la política (Ver Tabla 1), las variables utilizadas en la regresión cumplieron el supuesto de independencia respecto 
de la variable dependiente, atendiendo a que el valor del test de DurbinWatson fue aceptable $(d=1.86)$. Además, se descartaron problemas de colinealidad entre las variables usadas en cada ecuación, explicando la ecuación resultante el $21.8 \%$ de la varianza de la variable dependiente. Los resultados mostraron un impacto positivo de la formación cívico-política en escuela ( $\beta=.089, p=.048)$, así como de la atención a política en medios tradicionales $(\beta=.170, p<.001) y$, sobre todo, la conversación sobre política en familia $(\beta=.294, p<.001)$. Sin embargo, no se registró ningún impacto de la atención a política a través de medios online. En este sentido, se puede concluir que el interés político se vio incrementado entre aquellos estudiantes de primaria que tendían a seguir más la política en medios tradicionales, a conversar sobre esos asuntos con su familia y que, también, recibían una mayor formación cívico-política en la escuela.

TABLA 1

Variables explicativas del interés en política y el conocimiento político factual

INTERÉS POLITICO

CONOCIMIENTO POLÍTICO

\begin{tabular}{|c|c|c|c|c|c|c|}
\hline & \multicolumn{3}{|c|}{$(\mathrm{n}=1,009)$} & \multicolumn{3}{|c|}{$(\mathrm{n}=1,009)$} \\
\hline & B & SE & $\beta$ & B & SE & $\beta$ \\
\hline Sexo del participante ( 1 = niña) & -.078 & .082 & -.040 & .220 & .232 & .043 \\
\hline Edad del participante & .116 & .074 & .083 & -.089 & .212 & -.024 \\
\hline Nivel de estudios de la madre & .043 & .041 & .057 & .199 & .118 & $.102+$ \\
\hline Nivel de estudios del padre & -.014 & .043 & -.018 & .122 & .124 & .060 \\
\hline Tipo de escuela ( 1 = privada) & -.012 & .166 & -.003 & .702 & .472 & .081 \\
\hline Área del estado (1 = no ZMM) & -.282 & .134 & $-.102^{*}$ & -.173 & .383 & -.024 \\
\hline Grado (1 = sexto grado) & .021 & .116 & .010 & .389 & .331 & .070 \\
\hline Atención en medios tradicionales & .179 & .056 & $.170^{* * *}$ & .442 & .158 & $.161^{* *}$ \\
\hline Atención en medios sociales & .015 & .047 & .017 & -.232 & .132 & $-.100+$ \\
\hline Conversación política en familia & .353 & .056 & $.294^{* * *}$ & -.120 & .161 & -.038 \\
\hline $\begin{array}{l}\text { Formación cívico-política en la } \\
\text { escuela }\end{array}$ & .108 & .054 & $.089 *$ & -.005 & .154 & -.001 \\
\hline$R^{2}$ & & $21.8 \%$ & & & $4.9 \%$ & \\
\hline$d$ & & 1.86 & & & 1.92 & \\
\hline
\end{tabular}

Nota: ZMM = Zona Metropolitana de Monterrey. ${ }^{*} \mathrm{p}<.05 ;{ }^{* *} \mathrm{p}<.01 ;{ }^{* * *} \mathrm{p}<.001$ Fuente: elaboración propia.

Por su parte, el modelo explicativo del conocimiento político factual también resultó estadísticamente significativo, si bien sólo consiguió explicar el 4.9\% de la varianza de la variable dependiente (VerTabla 1). En cuanto a las variables utilizadas en la regresión, todas cumplieron el supuesto de independencia respecto de la variable dependiente $(\mathrm{d}=1.92)$ y no se encontraron problemas de colinealidad entre las mismas. Atendiendo a los resultados obtenidos, se observó que únicamente la atención a política en medios tradicionales $(\beta=.161, p=.005)$ tuvo un impacto positivo en el aumento del nivel de conocimiento político factual. Cabe mencionar además que la atención a política a través de medios online presentó un impacto tendencial $(\beta=-.100$, $p=.080$ ). En este sentido, un aumento en el uso de estos medios tenía una tendencia a disminuir el conocimiento mantenido por los participantes en 
el estudio. Por su parte, ni la conversación política en familia y la formación cívico-política en escuela lograron impactar en el conocimiento político factual de los estudiantes.

En cuanto a la ecuación calculada para explicar el sentimiento de eficacia política interna de los estudiantes encuestados, el modelo obtenido explicó el $19 \%$ de la varianza de la variable dependiente (VerTabla 2). Asimismo, y como se detectó en las anteriores regresiones, las variables utilizadas cumplieron el supuesto de independencia respecto de la variable dependiente $(\mathrm{d}=$ 2.05) y no presentaron colinealidad en las relaciones entre ellas. Como se puede observar en los resultados obtenidos, la atención a política en medios tradicionales ( $\beta=.180, p=.002$ ) volvió a emerger como variable explicativa del incremento de la actitud estudiada. Además, también la conversación política en familia impactó de forma positiva, si bien en un nivel inferior ( $\beta$ $=.129, \mathrm{p}=.012$ ). Finalmente, la formación cívico-política adquirida en la escuela destacó como la principal variable explicativa de la eficacia política interna de los estudiantes $(\beta=.233, p<.001)$. Sin embargo, no se encontró ningún impacto de la atención a política a través de medios online. Por tanto, se puede concluir que la eficacia política interna de los estudiantes se vio incrementada entre quienes tendían a seguir más la política en medios tradicionales, a conversar sobre esos asuntos con su familia y que, también, recibían una mayor formación cívico-política en la escuela.

TABLA 2

Variables explicativas del sentimiento de eficacia política interna y externa

\begin{tabular}{|c|c|c|c|c|c|c|}
\hline & \multirow{2}{*}{\multicolumn{3}{|c|}{$\begin{array}{l}\text { EFICACIA POLÍTICA } \\
\text { INTERNA } \\
(\mathrm{n}=1,009)\end{array}$}} & \multirow{2}{*}{\multicolumn{3}{|c|}{$\begin{array}{c}\text { EFICACIA POLÍTICA } \\
\text { EXTERNA } \\
(\mathrm{n}=1,009)\end{array}$}} \\
\hline & & & & & & \\
\hline & B & SE & $\beta$ & $\mathrm{B}$ & SE & $\beta$ \\
\hline Sexo del participante (1 = niña) & -.117 & .066 & -.080 & -.179 & .052 & $-.154 * * *$ \\
\hline Edad del participante & .037 & .061 & .035 & -.003 & .048 & -.004 \\
\hline Nivel de estudios de la madre & .054 & .034 & .096 & .013 & .027 & .029 \\
\hline Nivel de estudios del padre & .020 & .035 & .035 & .074 & .028 & $.160^{* *}$ \\
\hline Tipo de escuela ( 1 = privada) & -.081 & .136 & -.032 & -.115 & .106 & -.058 \\
\hline Área del estado (1 = no ZMM) & .099 & .110 & .048 & .033 & .086 & .020 \\
\hline Grado ( 1 = sexto grado) & -.063 & .095 & -.040 & .050 & .074 & .039 \\
\hline Atención en medios tradicionales & .141 & .045 & $.180^{* *}$ & .010 & .036 & .016 \\
\hline Atención en medios sociales & .003 & .038 & .005 & .004 & .030 & .007 \\
\hline Conversación política en familia & .119 & .047 & $.129 *$ & .014 & .036 & .019 \\
\hline $\begin{array}{l}\text { Formación cívico-política en la } \\
\text { escuela }\end{array}$ & .208 & .043 & $.233^{* * *}$ & .161 & .034 & $.226^{* * *}$ \\
\hline$R^{2}$ & & $19 \%$ & & & $12.6 \%$ & \\
\hline$d$ & & 2.05 & & & 1.98 & \\
\hline
\end{tabular}


En último lugar, se analizaron las variables explicativas del sentimiento de eficacia política externa mantenido de los participantes en el estudio (Ver Tabla 2). Las variables utilizadas cumplieron el supuesto de independencia respecto de la variable dependiente $(d=1.98)$, consiguiendo explicar en conjunto el $12.6 \%$ de la varianza de la variable dependiente. Los resultados obtenidos arrojaron un modelo explicativo más reducido, manifestándose únicamente la formación cívico-política adquirida en la escuela como agente socializador con capacidad de explicar de forma positiva el incremento de la eficacia política externa $(\beta=.226, p<.001)$. Ello lleva a concluir que la eficacia política externa tendía a incrementarse únicamente entre los estudiantes de primaria que recibían una mayor formación cívico-política en la escuela, sin que ningún otro agente socializador llegara a influir en esta orientación política.

\section{Discusión y conclusiones}

En el presente artículo se planteó como objetivo determinar el peso que cada uno de los principales agentes socializadores -escuela, familia y medios de comunicación- tienen en el desarrollo de ciertas orientaciones constitutivas de la cultura política mantenida por los estudiantes de primaria en el caso del estado mexicano de Nuevo León, como son el interés y conocimiento políticos, así como el sentimiento de eficacia política interna y externa. Para ello, se plantearon cuatro preguntas de investigación, relativas a la explicación de cada orientación política. Además, se establecieron indicadores específicos para medir el papel desarrollado por los diferentes agentes socializadores: se midió el grado de atención prestada por los estudiantes, ellos solos o en compañía de sus familiares, a contenidos de política en medios tradicionales y/o en medios sociales, el nivel de conversación política realizada con los miembros de la familia y el nivel de formación cívico-política recibida en la escuela (Ver Tabla 3), cuyos resultados e implicaciones se discuten a continuación.

TABLA 3

Tabla resumen de los modelos estudiados

\begin{tabular}{l|cc|cc}
\hline & $\begin{array}{l}\text { INTERÉS } \\
\text { POLÍTICO }\end{array}$ & $\begin{array}{c}\text { CONOCIMIENTO } \\
\text { POLÍTICO }\end{array}$ & $\begin{array}{l}\text { EFICACIA } \\
\text { POLÍTICA } \\
\text { INTERNA }\end{array}$ & $\begin{array}{c}\text { EFICACIA } \\
\text { POLITICA } \\
\text { EXTERNA }\end{array}$ \\
\hline $\begin{array}{l}\text { Atención en medios } \\
\text { tradicionales } \\
\begin{array}{l}\text { Atención en medios } \\
\text { sociales }\end{array}\end{array}$ & + & + & + & \\
$\begin{array}{l}\text { Conversación política en } \\
\text { familia }\end{array}$ & + & & + & \\
$\begin{array}{l}\text { Formación cívico- } \\
\text { política en escuela }\end{array}$ & + & + & +
\end{tabular}

Nota: Se marcan con un signo de + las variables que impactaron de forma significativa y positiva en cada componente de la cultura política.

Fuente: elaboración propia. 
Respecto a la primera pregunta de investigación, el artículo cuestionó en qué medida impactaron cada uno de los agentes socializadores en el desarrollo de interés hacia la política por parte de los estudiantes de primaria. Los resultados permiten determinar que, salvo la atención a contenidos de política en medios sociales, todos los demás agentes contribuyen, en mayor o menos medida, al desarrollo de un compromiso afectivo o interés político de los estudiantes con la política (Ver Tabla 3). En concreto, es la atención a política en contenidos de los medios tradicionales $y$, sobre todo, la familia a través del mantenimiento de actividades de dialogo y conversación los dos agentes que en mayor medida contribuyen al incremento de esta orientación previa o motivación hacia la política. Se observa, por tanto, cómo a pesar del avance en el desarrollo de nuevos medios de transmisión de información, como pueden suponer los medios sociales que se han observado como generadores de interés político en otros contextos (Kruikemeier y Shehata, 2017), los mecanismos tradicionales de comunicación y transmisión de información, en particular las redes familiares, siguen siendo el principal intermediario para incentivar el interés de los niños por los asuntos políticos, tal y como otros autores detectaron hace varios años (Huerta y García, 2008; Mayer y Schmidt, 2004).

En cuanto a la segunda pregunta de investigación, ésta se planteaba determinar en qué medida cada agente socializador impactó sobre el conocimiento político mantenido por los participantes en el estudio. El análisis revela una influencia significativa de ambas variables mediáticas, en particular de la atención a contenidos de política en medios de comunicación tradicionales. Es decir, se observa que el seguimiento de noticias, de programas en medios de política, así como de sátira política contribuyen a aumentar el compromiso cognitivo de los estudiantes. Sin embargo, el seguimiento o atención a política en nuevos medios o medios sociales más bien contribuye a una disminución de este compromiso, si bien su influencia es menor a la otra atención registrada. Se observa, por tanto, una influencia diferenciada en función del tipo de medio, algo interesante atendiendo a la disparidad en el consumo de estos medios entre jóvenes. Así, los medios tradicionales, a pesar de ceder en uso entre los jóvenes (Echeverría y Meyer, 2017), sin embargo, cumplen un rol más influyente a la hora de configurar un compromiso político entre quienes los consumen, como se ha puesto de manifiesto en varios estudios previos (Huerta et al., 2006; Tapia, 2003).

Por su parte, el seguimiento de política en medios online tiene un impacto más moderado en el nivel de conocimiento político. Sin embargo, y aunque su nivel de influencia fuera únicamente tendencial, no deja de constituir un dato significativo, pues se aleja de lo observado en estudios previos. Se concluye, por tanto, que los nuevos medios no logran acrecentar el compromiso cognitivo de los futuros ciudadanos, sino más bien hacen que éste disminuya, aun cuando sean utilizados para informarse de política. Un resultado contrario a lo encontrado acerca de la influencia de este tipo de consumo para informarse de política en otros contextos (Muralidharan y Sung, 2016), donde habitualmente los medios y redes sociales contribuyen al involucramiento del estudiante a un nivel cognitivo. Este resultado abre, por tanto, un interesante debate acerca del verdadero papel que los nuevos medios están cumpliendo en la configuración de la cultura política de los futuros ciudadanos. Un debate en el que no pueden obviarse los verdaderos motivos de uso de estos nuevos medios -como mecanismo de información o más bien de entretenimiento-, así como el estudio de los contenidos que circulan en la red y su contribución al aprendizaje político. 
Por su parte, la tercera pregunta de investigación planteada se cuestionaba el impacto ejercido por los diferentes actores sobre la eficacia política interna de los participantes. Al respecto, los resultados ponen de manifiesto una influencia positiva de los hábitos conversacionales realizados en familia, así como del consumo de política realizado a través de los medios tradicionales, como la prensa, radio o televisión. Es decir, la familia y los medios tradicionales parecen contribuir a incrementar el sentimiento de capacitación para participar en política de los estudiantes. Este hallazgo concuerda con los obtenidos previamente en otros contextos, como los del estudio de Lee et al. (2012) o el más reciente de Arens y Watermann, 2017, que ponen de manifiesto cómo el consumo de contenidos políticos en los medios de comunicación tiene un peso crucial en la generación de compromiso democrático entre los jóvenes. Un compromiso que, en buena medida, puede llegar a desarrollarse en el individuo en la medida en que éste se perciba como un ciudadano formado y con capacidad de influir en la esfera pública para cambiar al sistema, a través del desarrollo de procesos de participación política y cívica.

Pero, más allá de la influencia realizada por los medios, fue la escuela a través de sus procesos de formación cívico-política el agente que en mayor medida contribuye al incremento de este sentimiento de eficacia política interna de los estudiantes. Un papel también desarrollado, en línea con lo planteado en la cuarta pregunta de investigación, con respecto al desarrollo de eficacia política externa. Al respecto, la escuela es el único factor que hace aumentar esta sensación de ser tenidos en cuenta por el sistema y los actores de autoridad respecto a las demandas que como ciudadanos puedan tener (Arens y Watermann, 2017). Este papel jugado por la escuela a través de sus procesos de formación cívica se vuelve crucial, pues es una institución que puede favorecer el desarrollo de sentimientos favorables hacia la democracia a través de la generación de mecanismos de participación de sus estudiantes (Imhoff y Brussino, 2013; Stojnic, 2015). Sobre todo, cuando en diferentes ocasiones se ha detectado cómo la percepción hacia la política de los estudiantes de primaria es más bien negativa (Huerta et al., 2006; Huerta y García, 2008), con una clara manifestación de actitudes desafectas a nivel político (Fernández Poncela, 2009; Morales, 2014).

En términos generales, los resultados obtenidos permiten posicionar a la escuela como un agente socializador crucial para el desarrollo de las orientaciones vinculadas con el involucramiento democrático de los niños con el sistema, lo cual se asume que repercutirá en sus niveles de compromiso político futuros una vez que se conviertan en ciudadanos con plenos derechos y deberes. Ahora bien, aunque la escuela es el agente que en mayor medida contribuye al cambio en las actitudes de los estudiantes de primaria, no consigue impactar, sorprendentemente, en un cambio a nivel cognitivo en el conocimiento político mantenido por estos estudiantes. Esto a pesar de que se podría considerar como un agente clave para el aprendizaje de los conocimientos que configuran la cultura política de la sociedad. $\mathrm{Ni}$ siquiera se observa esta aportación del sistema educativo en conjunto al desarrollado por la atención a política en medios tradicionales, que como se recordará fue la única variable explicativa del modelo. Ello lleva a la necesidad de plantear un debate acerca de los contenidos políticos y las prácticas educativas desarrolladas para su aprendizaje, atendiendo al hecho de que no parecen contribuir a un compromiso cognitivo adecuado de sus estudiantes. Así como de la ya analizada influencia que el modelo de educación pública, frente a la privada, tiene en la formación ciudadana (Huerta y García, 2008). 
Abundando en este debate, pareciera que el impacto de la escuela se produce en mayor medida de una forma indirecta, a través del desarrollo de una mayor eficacia política interna y externa del estudiante, que de una forma directa con el incremento del compromiso cognitivo. En todo caso, esta labor realizada por la escuela es digna de ser señalada, pues puede derivar en un mayor compromiso político efectivo del estudiante y futuro ciudadano, en tanto la relación entre eficacia y participación políticas está claramente demostrada (Arens y Watermann, 2017), de tal manera que entre aquellos ciudadanos que se presenta un mayor sentimiento de tener una buena formación política y de poder contribuir con calidad al sistema, mayor es también el nivel en el que participan efectivamente en las diferentes actividades que el sistema les ofrece. Es en el entorno educativo, por tanto, donde el estudiante aprenderá los valores democráticos y lo que implica involucrarse con la democracia para tratar de incidir en los actos de gobierno. Una línea de investigación que debe seguir profundizándose a través de estudios que permitan determinar cómo incide esta formación en la composición del ideario político del estudiante.

Por su parte, la atención a política en contenidos transmitidos por medios tradicionales conserva un papel predominante en la configuración de la cultura política de los estudiantes de primaria. A pesar de la fuerte presencia que, como se sabe, tienen los medios sociales en el entorno actual, en particular entre los grupos de menor edad (Echeverría, y Meyer, 2017; Kruikemeier y Shehata, 2017), las noticias a través de medios tradicionales y programas de política parecen seguir incidiendo fuertemente en el interés $y$, sobre todo, en el conocimiento político de los estudiantes. No en vano, este agente es el único que logró impactar y producir un incremento del aprendizaje político de los participantes, contribuyendo así a que los esquemas que sobre política mantengan los estudiantes se acrecienten y profundicen. Un ejercicio de socialización ya detectado en estudios previos (Huerta et al., 2006; Huerta y García, 2008), sobre todo en relación con el peso de la televisión en la generación de cultura política. Es necesario abrir un debate sobre el verdadero poder de impacto de estos contenidos mediáticos y cómo hacer que éste se incremente, posiblemente a través de la elaboración de contenidos más atractivos que consigan atraer la atención de la población objeto de este estudio.

Los resultados del estudio permiten vislumbrar, además, cómo la familia sigue siendo un entorno prioritario donde se produce el primer y más importante acercamiento de los niños con la política. En concreto, el desarrollo de una adecuada conversación política con los diferentes integrantes del núcleo familiar constituye un factor clave para generar en estos pre-ciudadanos un adecuado compromiso afectivo, que se manifiesta en la presencia de un interés hacia lo que ocurre en el terreno político. Esta es una orientación previa que determina, en gran medida, la motivación de las personas por obtener información acerca de su entorno, lo que deriva en una mayor sofisticación política y, por consiguiente, en un mejor compromiso manifestado a través del involucramiento en la toma decisiones. Se observa, por tanto, la mancuerna importante que familia y escuela siguen constituyendo en la socialización de actitudes políticas del estudiante manifestadas en el terreno individual, pero también colectivo.

Finalmente, es reseñable destacar el casi nulo impacto que el consumo de política en medios y redes sociales parece tener sobre las orientaciones constitutivas de la cultura política en los estudiantes de primaria. A pesar de su papel predominante en la dieta mediática de las nuevas generaciones, 
parece que su consumo se sigue circunscribiendo hacia otros aspectos, siendo anecdótico el seguimiento de contenidos de política a través de estas vías digitales. Tanto, que ni siquiera entre quienes indicaron sí hacerlo, su presencia llega a impactar en las actitudes políticas estudiadas, salvo en el conocimiento político, aunque fuera de forma tendencial. Así, cabe destacar que la atención a política en estos nuevos medios impacta en un decrecimiento del conocimiento político de los participantes y, por tanto, en un menoscabo de su sofisticación política. Esto abre una necesaria línea de investigación acerca del uso político que los grupos de menor edad están haciendo de los medios sociales, así como de las repercusiones que su consumo tiene en el desarrollo de una mejor o peor cultura política de los futuros ciudadanos.

\section{Referencias}

Berger, P., y Thomas, L. (1983). La construcción de la realidad social. Buenos Aires, Argentina: Amorrortu Editores.

Chaffee, S. H., Ward, L. S., y Tipton, L. P. (1970). Mass communication and political socialization. Journalism Quarterly, 47(4), 647-659. doi: $10.1177 / 107769907004700401$

Cullingford, C. (1992). Children and society. Children's attitudes to politics and power. Londres, RU: Casell.

Del Río, N. (2008). Jóvenes ciudadanos en la arena social. En Anuario de Investigación (pp. 116-140). Ciudad de México, México: UAN-X.

Delli Carpini, M. X. (2004). Mediating democratic engagement: The impact of communications on citizens' involvement in political and civic life. En L. L. Kaid (Ed.), Handbook of political communication (pp. 395-434). Nueva York, NY, EU: Lawrence Erlbaum. 
Dahl, V., y Abdelzadeh, A. (2017). Self-selection or socialization? The longitudinal relation between civic engagement and political orientations among adolescents. Nonprofit and Voluntary Sector Quarterly, 46(6), 1250-1269. doi: 10.1177/0899764017728363

Echeverría, M., y Meyer, J. A. (2017). Internet y socialización política. Consecuencias en la participación juvenil. Anagramas, 15(30), 29-50. doi: 10.22395/angr.v15n30a1

Eveland, W. P., McLeod, J. M., y Horowitz, E. M. (1998). Communication and age in childhood political socialization: An interactive model of political development. Journalism \& Mass Communication Quarterly, 75(4), 699-718. doi: 10.1177/107769909807500406

Fernández Poncela, A. M. (2009). España-México: democracia, interés político y asociacionismo juvenil. El Cotidiano, (155), 115-120.

Huerta, E., Bañuelos, B., Rodríguez, A., Luz, S., y Gómez, C. (2006). El rol de la televisión en la socialización política de los niños: resultados preliminares. UNIrevista, 1(3), 1-13.

Huerta, W., y García, E. (2008). La formación del ciudadano: El papel de la televisión y la comunicación en la socialización política. Comunicación y Sociedad, (10), 163-189.

Igartua, J. J. (2006). Métodos cuantitativos de investigación en comunicación. Barcelona, España: Bosch.

Imhoff, D., y Brussino, S. (2013). Participación sociopolítica infantil y procesos de socialización política: exploración con niños y niñas de la ciudad de córdoba, Argentina. Liberabit, 19(2), 205-213.

Kruikemeier, S., y Shehata, A. (2017). News media use and political engagement among adolescents: An analysis of virtuous circles using panel data. Political Communication, 34(2), 221-242. doi: 10.1080/10584609.2016.1174760 
Lee, N.-J., Shah, D. V., y McLeod, M. J. (2012). Processes of political socialization: A communication mediation approach to youth civic engagement. Communication Research, 40(5), 669-697. doi: $10.1177 / 0093650212436712$

López Gómez, G. (2003). Televisión y socialización política de los niños en Monterrey y Juchitán (Tesis de Maestría). Tecnológico de Monterrey, México.

Mayer, J. D., y Schmidt, H. M. (2004). Gendered political socialization in four contexts: political interest and values among junior high school students in China, Japan, Mexico, and the United States. The Social Science Journal, 41(3), 393-407. doi: 10.1016/j.soscij.2004.04.024

Miron, D., y Bryant, J. (2007). Mass media and voter turnout. En R. W. Preiss, B. M. Gayle, N. Burrell, M. Allen y J. Bryant (Eds.), Mass media research: Advances through metaanalysis (pp. 391-413). Mahwah, NJ, EU: Lawrence Erlbaum Associates.

Morales, P. G. (2014). Percepciones sobre la socialización política en los adolescentes: El papel de la escuela, la familia y los medios de comunicación. Tesis de maestría, Universidad Autónoma de Nuevo León, México.

Muralidharan, S., y Sung, Y.(2016). Direct and mediating effects of information efficacy on voting behavior: political socialization of young adults in the 2012 US presidential election. Communication Reports, 29(2), 100 114. doi: $10.1080 / 08934215.2015 .1064537$

Nateras, J. (2007). Información política en estudiantes de primaria y secundaria. Revista Mexicana de Investiación Educativa, 12(35) 13571403.

Prior, M. (2010). You've either got it or you don't? The stability of political interest over the life cycle. The Journal of Politics, 72(3), 747-766. doi: $10.1017 /$ S0022381610000149 
Rodríguez, A., y Muñiz, C. (2009). Recepción de la información política televisada en niños regiomontanos durante la campaña presidencial del 2006. Comunicación y Sociedad, (11), 99-134.

Sears, D. O., y Valentino, N. A. (1997). Politics matters: Political events as catalysts for preadult socialization. American Political Science Review, 91(01), 45-65. doi: 10.2307/2952258

Segovia, R. (1975). La socialización política del niño mexicano. Ciudad de México, México: COLMEX.

Segovia, R. (2001). La politización del niño mexicano. Ciudad de México, México: COLMEX.

Stojnic, L. G. (2015). El efecto de la experiencia escolar en el desarrollo de actitudes favorables hacia la democracia como mejor sistema político: el caso de estudiantes peruanos recién graduados del nivel secundario. Colombia Internacional, (85), 111-139. doi: 10.7440/ colombiaint85.2015.04

Tapia, E. (2003). Socialización Política y educación cívica en los niños. Querétaro, México: Instituto Mora. 


\section{e-Ciencias de la Información}
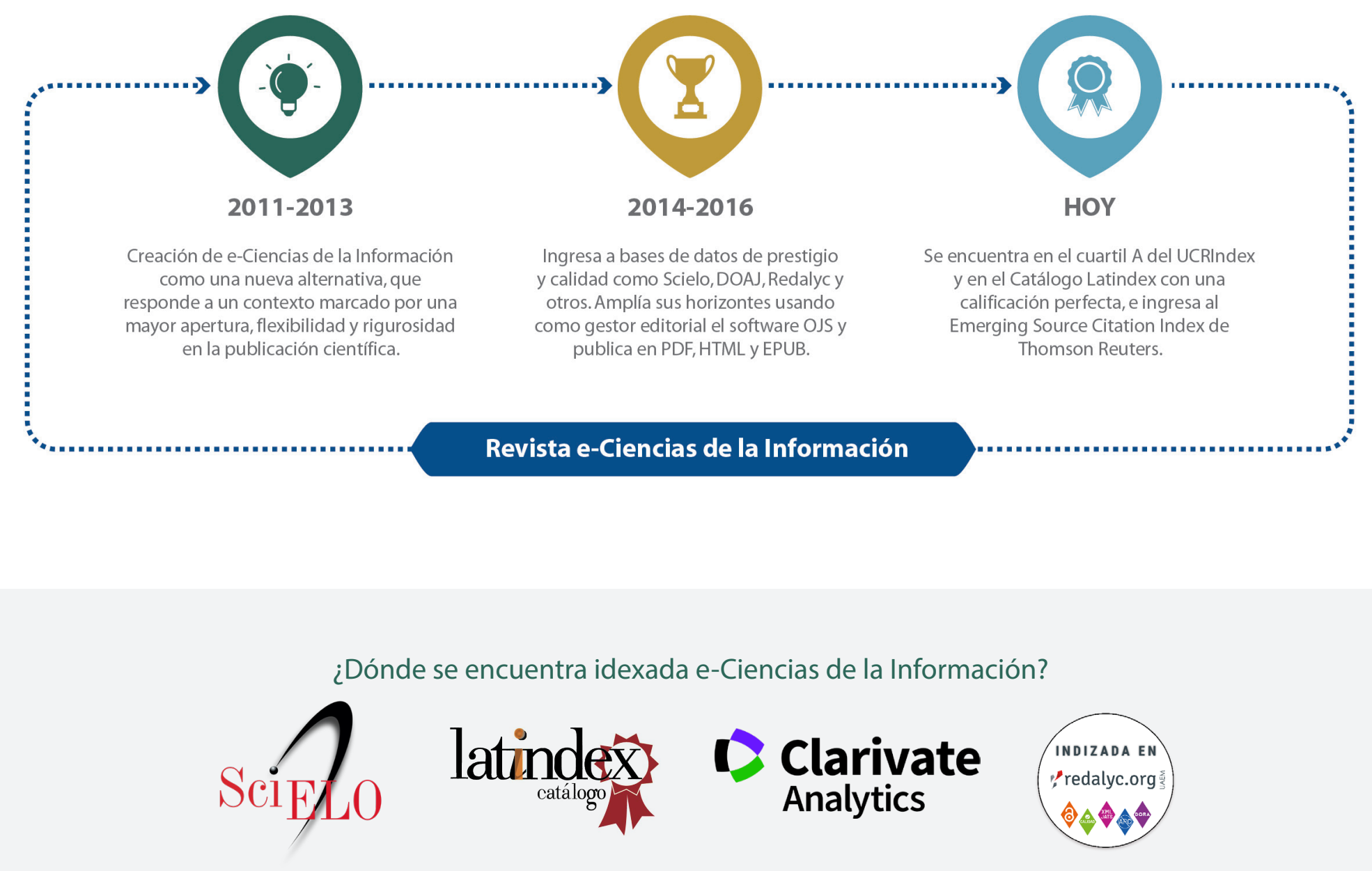

Para más información ingrese a nuestra lista completa de indexadores

¿Desea publicar su trabajo?

Ingrese aquí

O escríbanos a la siguiente dirección revista.ebci@ucr.ac.cr 